http://jmscr.igmpublication.org/home/ ISSN (e)-2347-176x ISSN (p) 2455-0450 crossref DOI: https://dx.doi.org/10.18535/jmscr/v8i3.70

\title{
To study the association of sudden sensorineural hearing loss and mean platelet volume including other platelet parameters within an Indian population
}

\author{
Authors
}

\section{Ibadaiamon Mawroh $^{1 *}$, Kanwar Sen ${ }^{2}$, Sadhna Marwah $^{3}$}

${ }^{1}$ Department of Otorhinolaryngology, Head and Neck surgery, PGIMER, Dr Ram Manohar Lohia Hospital, New Delhi -110001, India

${ }^{2}$ Department of Otorhinolaryngology, Head and Neck surgery, PGIMER , Dr Ram Manohar Lohia Hospital, New Delhi- 110001, India

${ }^{3}$ Department of Pathology, PGIMER, Dr Ram Manohar Lohia Hospital, New Delhi- 110001, India *Corresponding Author

Ibadaiamon Mawroh

\begin{abstract}
Objective: In this study, we aim to determine whether patients with sudden SNHL in North India, had an elevated Mean platelet volume (MPV), platelet count (PC) and platelet distribution width (PDW).

Study Design: Cross- sectional observation study

Setting: PGIMER, Dr Ram Manohar Lohia Hospital, New Delhi

Subjects and Methods: In this study, a group of 30 patients and 30 healthy subjects were taken as controls. Blood samples were drawn immediately on admission. The platelet parameters were analysed in the Medonic M series haematology cell counter. Comparisons of the blood indices were made between the two groups. Quantitative variables were compared using Unpaired t-test /Mann Whitney test (when data sets were not normally distributed) between the two groups.

Qualitative variables were correlated using Chi Square test/Fischer's exact test. A p value of $<0.05$ was considered statistically significant.

Results: Although the MPV, PDW and PC were marginally higher in the cases compared to the control group, a significant difference $(p<0.05)$ was not observed between the two groups. The mean platelet volume showed a $p$ value of 0.197 , the platelet count had a $p$ value of 0.433 and the platelet distribution width revealed a p value of 0.67 .

Conclusion: Hence, the mean platelet volume and other platelet parameters are not associating factors in the occurrence of sudden sensorineural hearing loss.

Keywords: Mean platelet volume, platelet parameters, sudden sensorineural hearing loss.
\end{abstract}

\section{Introduction}

Idiopathic sudden SNHL is a common clinical syndrome that frequently poses a diagnostic and therapeutic dilemma because of uncertainty about causation, appropriate treatment, and prognosis in most instances. According to the national institute of deafness and other communication disorders (NIDCD), the most frequently diagnostic criteria is a decrease in hearing of greater than or equal to 30 decibels, affecting at least 3 consecutive 
frequencies within 72 hours. It is often described by the patient as an acute and rapid onset of subjective sensation of hearing impairment in one or both ears occurring within a period of 72 hours or less. Some patients reported that the hearing loss was noted instantaneously in the morning. Others described that it occurred gradually over a period of hours to days. Up to one- third of patients have an upper respiratory prodome preceding sudden SNHL suggesting the role of viruses in the etiology of this disease. Hearing loss is usually unilateral and is frequency accompanied by tinnitus in $70 \%$ of cases, vertigo or milder sensation of spatial disorientation in $50 \%$ of cases. Nearly all cases are unilateral; less than $2 \%$ of patients have bilateral involvement and typically involvement is sequential. Hearing loss may be mild in a restricted frequency or it may be total. ${ }^{1}$

The alterations in microcirculation of cochlea have long been suggested to be a cause of sudden SNHL $^{2}$. The blood supply to cochlea is maintained by the labyrinthine artery, which has no collateral vasculature. It has been proposed that thrombosis or vasospasm of the labyrinthine artery or its sub divisions, leads to impaired perfusion of the cochlea. Alterations in the microcirculations of the cochlea maybe a cause of idiopathic sudden SNHL. ${ }^{3}$ These pathologies decrease the blood supply to the cochlea, thus reducing intracochlear oxygen levels. As cochlear structures are fairly susceptible to even brief episodes of hypoxia, this type of obstruction can lead to either a transient or permanent hearing loss in experimental models. Vascular disorders may cause sudden SNHL because of cochlear injury and dysfunction secondary to anoxia or hypoxia. ${ }^{4}$ The clinical picture of sudden SNHL is similar to that of vascular diseases such as myocardial infarction and cerebral stroke. Acute onset of unilateral symptoms reinforces the suggestion of an underlying vascular occlusion.

MPV is the most commonly used measure of platelet size, and is a potential marker of platelet reactivity. ${ }^{5}$ Large platelets contain a greater number of dense granules and are enzymatically and metabolically more active and thus, have greater prothrombotic potential. High MPV is associated with a variety of established risk factors, including cardiovascular and cerebrovascular disorders; and low grade inflammatory conditions are prone to arterial and venous thrombosis ${ }^{6}$. M Sagit et al reported an increase in MPV in patients with idiopathic sudden SNHL indirectly supporting the hypothesis of vascular impairment as a pathogenic factor in sudden $\mathrm{SNHL}^{3}$. Ulu et al found a significant increase in volume of circulating platelets in 40 patients in the past 6 years when compared to a group of 40 healthy individuals of identical age and gender ${ }^{7}$. Zajtchuk et al reported that 8 to 14 patients with sudden SNHL had laboratory evidence of hypercoagulable state manifesting as abnormal thrombin generation and increase platelet count ${ }^{8}$. High platelet reactivity correlates with platelet function and activation, whether measured as aggregation, thromboxane synthesis, beta thromboglobulin release, procoagulant function or adhesion molecule expansion ${ }^{8-12}$.

\section{Materials and Methods}

A total of 60 subjects were included in the study of which 30 subjects were patients diagnosed with sudden SNHL and 30 healthy subjects were taken as controls. All patients having evidence of following disorders were excluded from the study:

- Sudden SNHL secondary to trauma

- Use of antithrombotic drugs or serotonin reuptake inhibitors

- Diabetes mellitus

- Hypertension

- Hypercholesterolaemia

- Arterial and venous thrombotic diseases

- Congestive heart failure

- Coronary artery disease

- Smokers

- Acute or chronic inflammatory diseases

- High body mass index

After diagnosis of sudden SNHL was made satisfying all inclusion and exclusion criteria, 
blood samples were drawn immediately anticoagulated with tri potassium ethylene diamine triacetic acid. Analysis were performed within two hours after phlebotomy in order to prevent in vitro platelet activation. MPV and other platelet parameters were measured using medonic M series haematology cell counter (3 part differential).

\section{Statistical Analysis}

Categorical variables were presented in number and percentage (\%) and continuous variables were presented as Mean \pm SD and Median. Normality of data was tested by Kolmogorov Smirnov test. If the normality was rejected then non parametric test was used.

Statistical tests were applied as follows:

Quantitative variables were compared using Unpaired t-test Mann Whitney test (when data sets were not normally distributed) between the two groups.

Qualitative variables were correlated using Chi Square test/Fischer's exact test. A $p$ value of < 0.05 was considered statistically significant. The data was entered in MS EXCEL spreadsheet and analysis was done using Statistical Package for social sciences (SPSS) version 21.0.

\section{Results}

The male:female ratios of the two groups were 2:1 for cases and 1:1 for controls respectively. The difference in the mean age of participants of the two groups was not significant. Participants with idiopathic sudden SNHL were $56.23 \pm 8.58$ years of age, and those of the control group were $59.93 \pm 8.91$ years. The body mass indices were similar for both the groups. All demographic characteristics of study participants are presented in table 1(a) and table 1(b).

\begin{tabular}{|l|c|c|c|c|}
\hline Gender & Cases & Control & Total & $p$ value \\
\hline Female & 10 & 15 & 25 & 0.190 \\
& $(33.33 \%)$ & $(50.00 \%)$ & $(41.67 \%)$ & \\
\hline Male & 20 & 15 & 35 & \\
& $(66.67 \%)$ & $(50.00 \%)$ & $(58.33 \%)$ & \\
& 30 & 30 & 60 & \\
& $(100.00 \%)$ & $(100.00 \%)$ & $(100.00 \%)$ & \\
\hline
\end{tabular}

1(a)

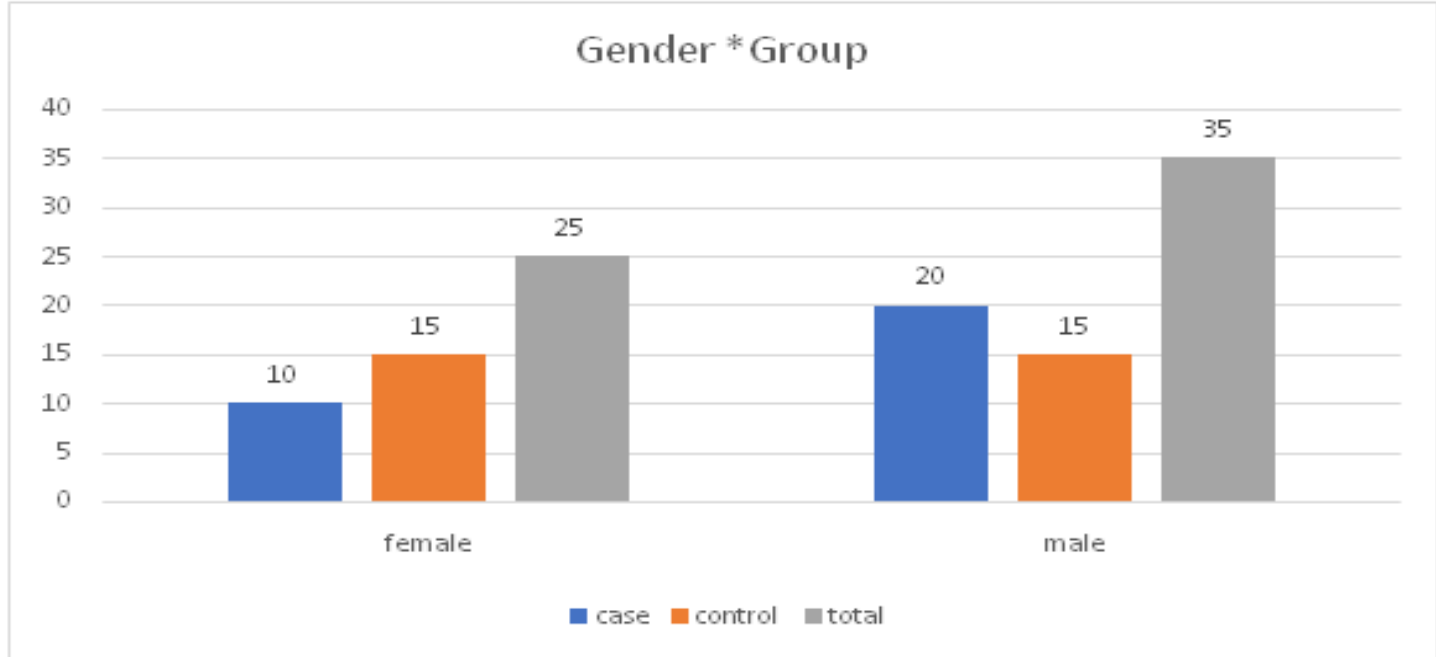

\begin{tabular}{|l|c|c|c|}
\hline Age & Case & Control & Total \\
\hline$<40$ & $6.67 \%$ & $13.33 \%$ & \multirow{2}{*}{60} \\
\cline { 1 - 3 } $41-50$ & $16.67 \%$ & $10 \%$ & \\
\cline { 1 - 3 } $51-60$ & $36.67 \%$ & $60 \%$ & \\
\cline { 1 - 2 }$>60$ & $40 \%$ & $16.67 \%$ & \\
\hline
\end{tabular}

$1(b)$ 


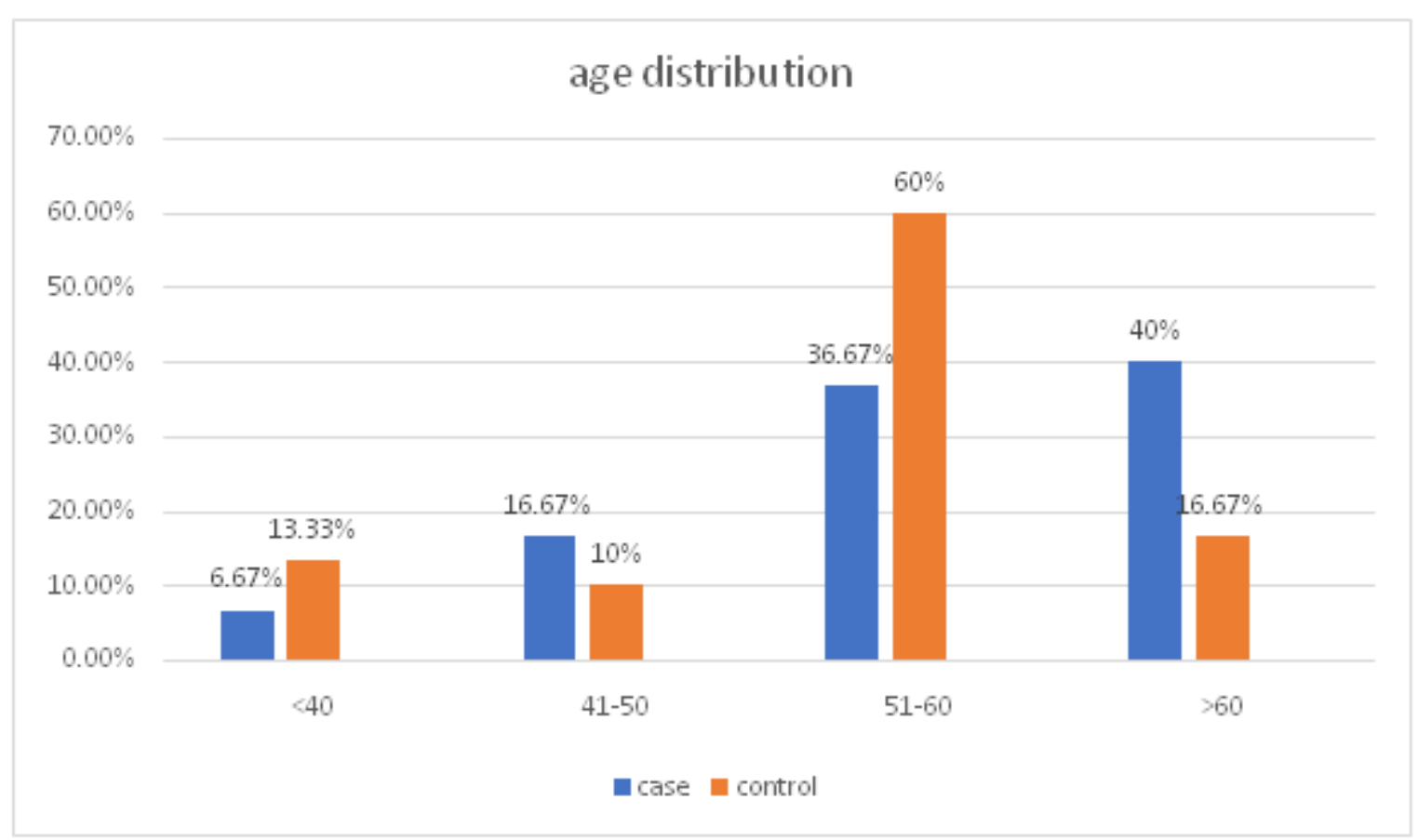

2(a) Mean Platelet Volume (fl)

\begin{tabular}{|l|c|c|l|}
\hline MPV(fl) & Case & Control & $p$ value \\
\hline Mean \pm SD & $9.37 \pm 1.57$ & $8.88 \pm 1.35$ & 0.197 \\
\cline { 1 - 3 } Median & 9.1 & 8.7 & \\
\cline { 1 - 3 } Min-Max & $7.2-13.3$ & $7-12.6$ & \\
\hline Inter Quartile range & $8-10.2$ & $8-9.2$ & \\
\hline
\end{tabular}

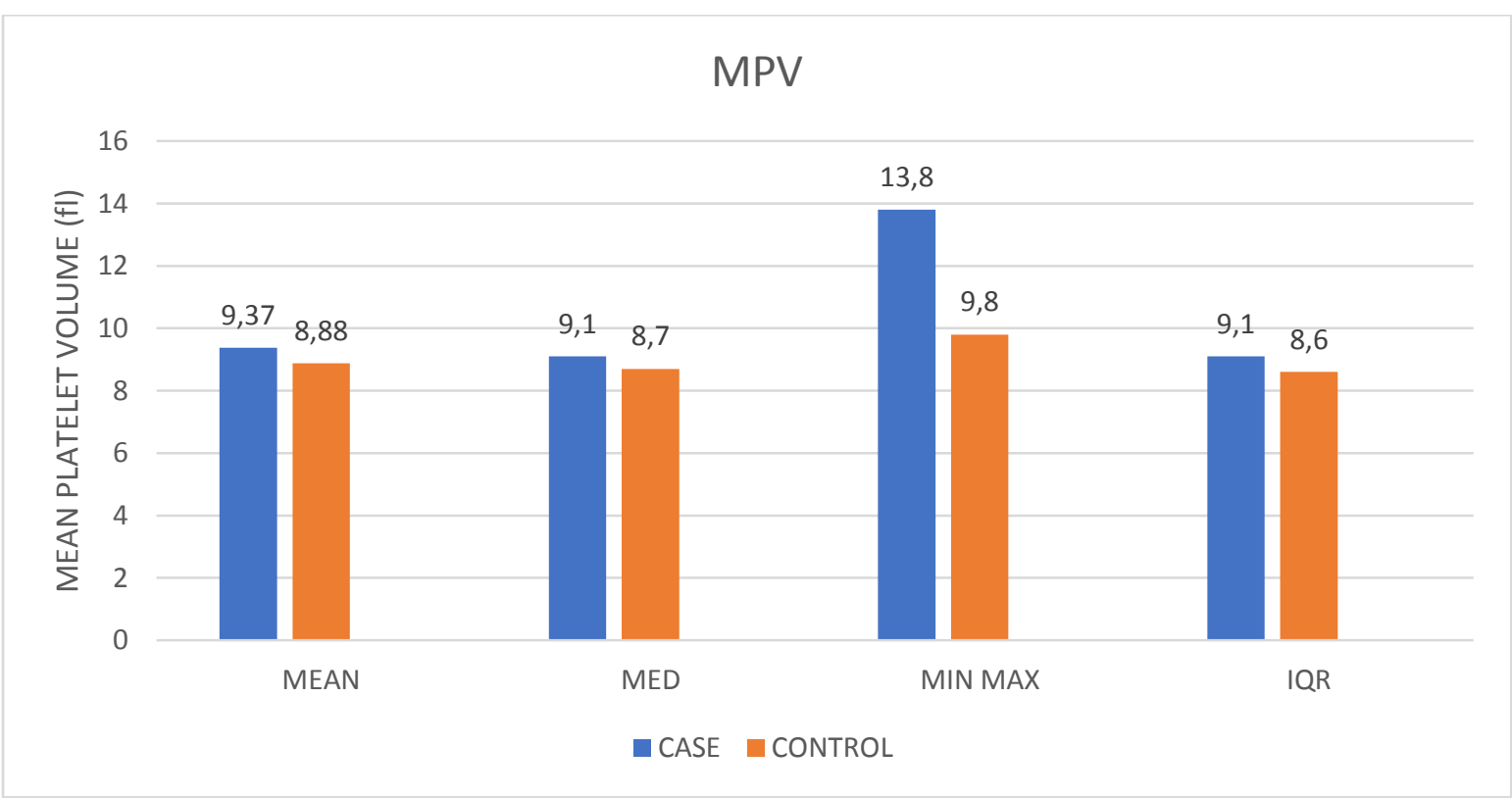

2(b) Platelet count $\left(\times 10^{3} / \mu 1\right)$

\begin{tabular}{|l|c|c|c|}
\hline $\mathrm{PC}\left(\mathrm{x} 10^{3} / \mu \mathrm{l}\right)$ & Case & control & $\mathrm{P}$ value \\
\hline Mean $\pm \mathrm{SD}$ & $189.6 \pm 69.34$ & $182.4 \pm 60.46$ & \multirow{2}{*}{0.433} \\
\cline { 1 - 3 } Median & 183.5 & 180 & \\
\cline { 1 - 3 } Min-max & $102-322$ & $90-300$ & \\
\hline Inner quartile range & $128-250$ & $136-220$ & \\
\hline
\end{tabular}




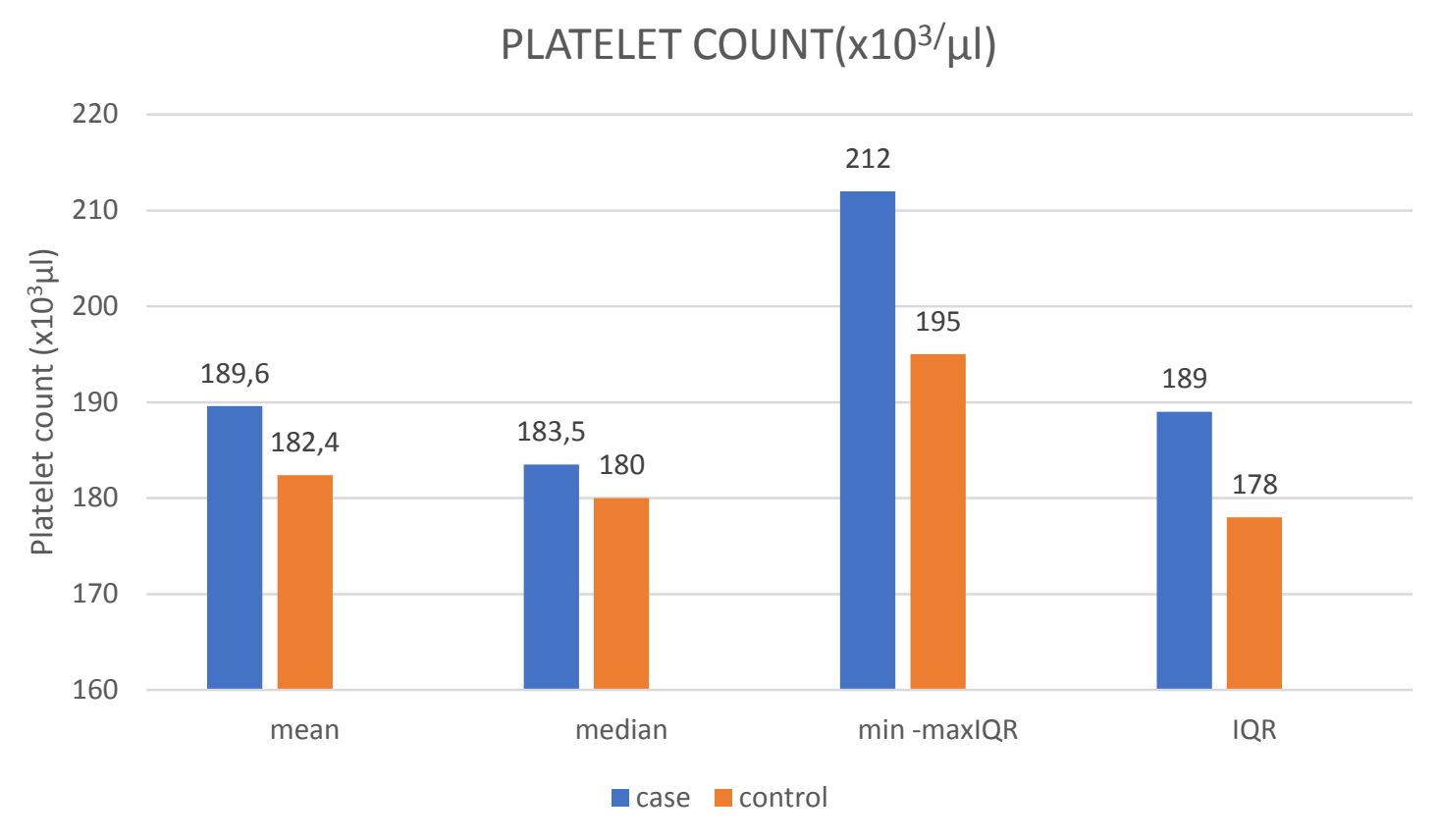

\begin{tabular}{|l|c|c|c|}
\hline PDW $(\%)$ & CASE & CONTROL & P value \\
\hline Mean \pm SD & $15.93 \pm 2.99$ & $15.42 \pm 3.07$ & \multirow{2}{*}{0.67} \\
\cline { 1 - 3 } Median & 15.1 & 14.1 & \\
\cline { 1 - 3 } Min-max & $12-20.5$ & $11.2-21$ & \\
\cline { 1 - 3 } Inner quartile range & $13.6-19$ & $13-19$ & \\
\hline
\end{tabular}

2(c) Platelet Distribution Width (\%)

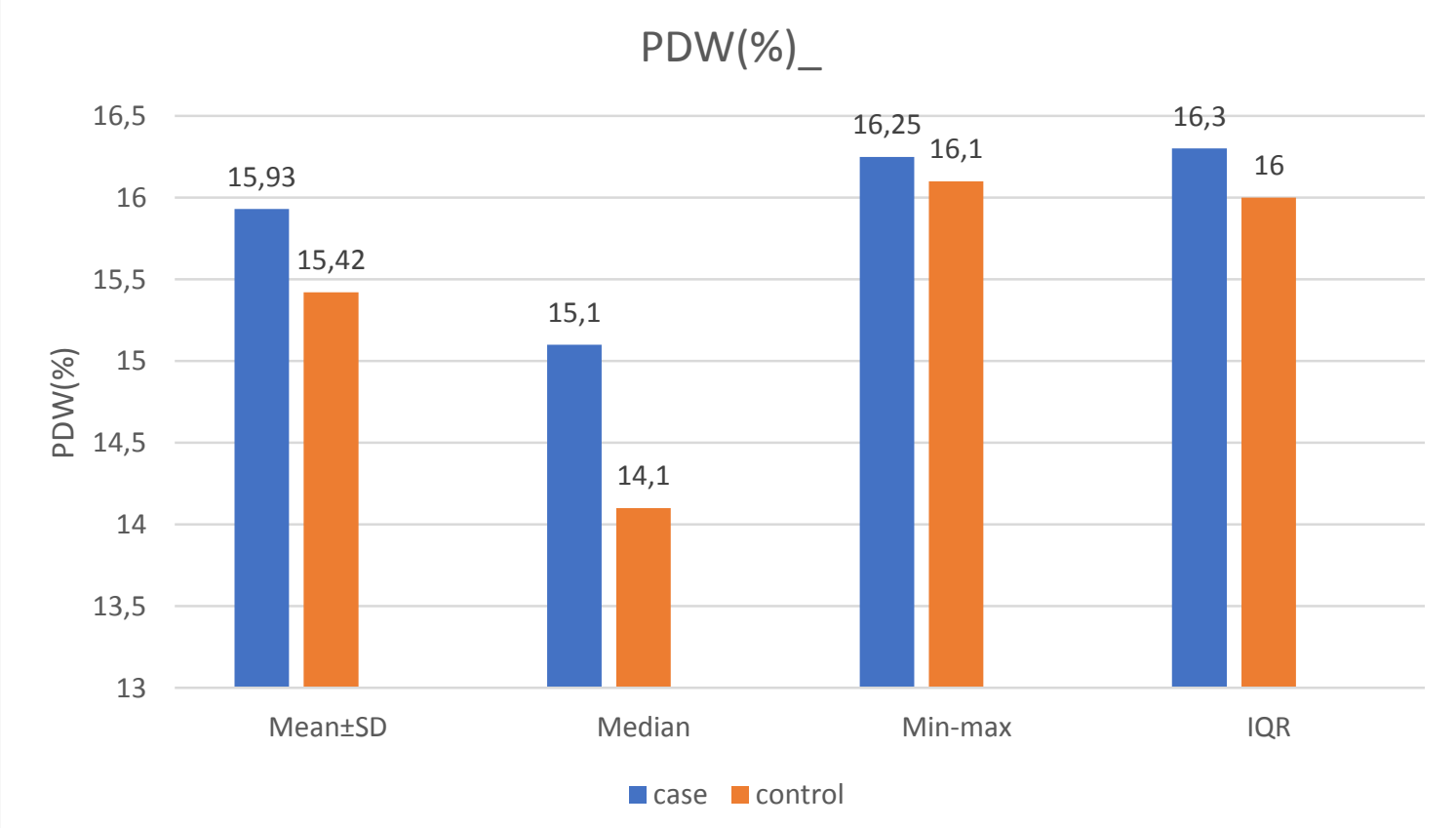



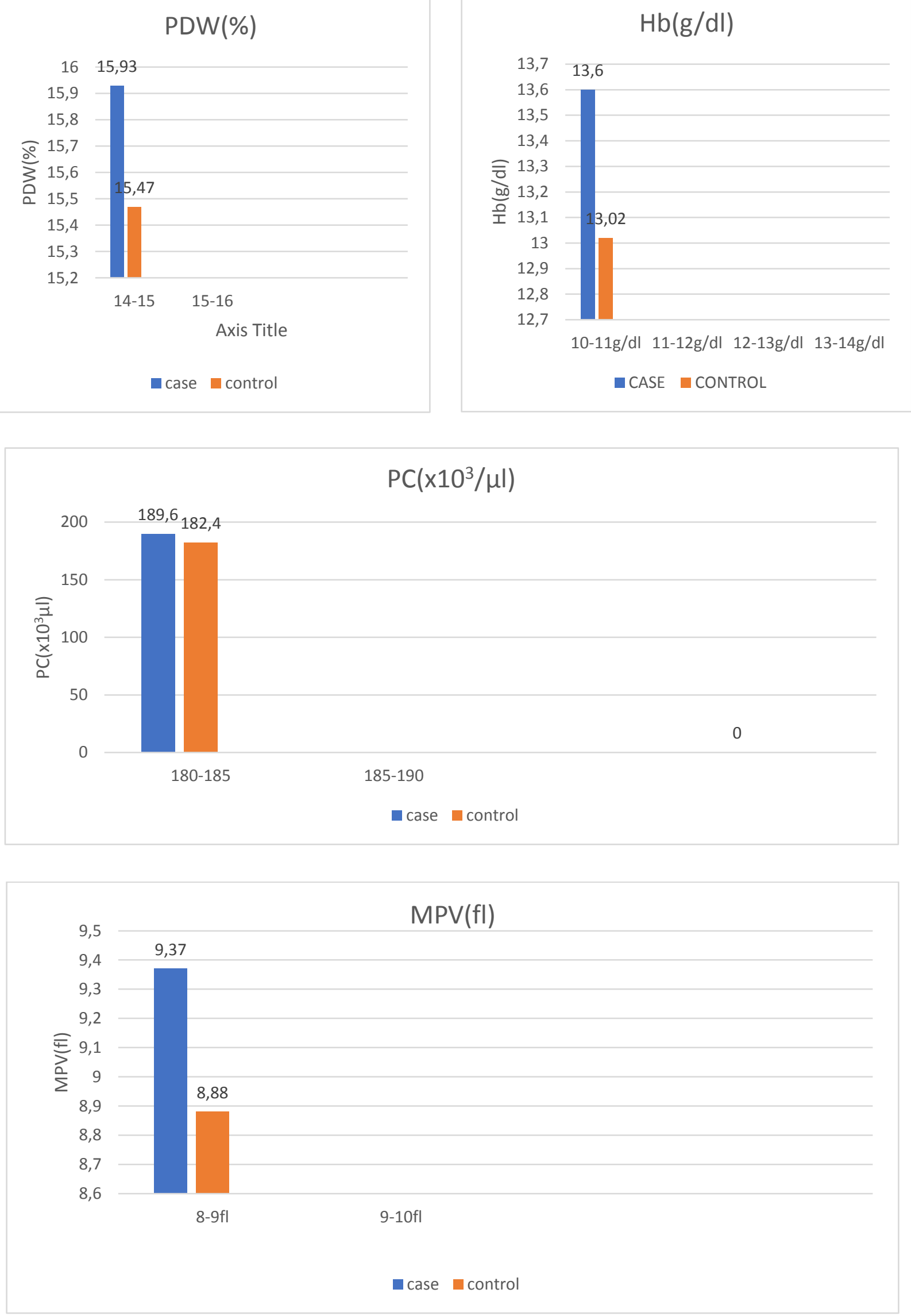

2(d) Summary of blood indices 


\section{Discussion}

Evaluation and management of sudden SNHL should be considered as a medical urgency if not emergency $^{13}$. Sudden SNHL is a common otological disorder with an increasing rate of incidence worldwide, based on current reports. Sudden SNHL requires immediate attention due to its varied etiologic factors and prognostic features. Although in most cases, the severity of disease is mild, it can lead tolong term hearing loss, permanent hearing loss and conditions such as tinnitus. In this study, one of the most commonly cited putative causes of sudden SNHL was considered; which is vascular disturbance. The tympanic membrane appears undamaged at otoscopy. The audiometric examination is important to emphasize the degree and type of hearing loss. In accurate literature revision, most accredited causes of sudden SNHL appears to be viral diseases, vascular sclerosis of microcirculation of cochlea, temporary breaks of the inner ear membranes and immune mediated reactions.

The vascular disease theories of sudden SNHL describe a spectrum of pathophysiological alterations at the capillary and microvascular levels.They include embolism, blood sludging, hypercoagulability, vasospasm, arteriosclerosis, systemic vascular disease and connective tissue disorders $^{9-12}$. It has been proposed that thrombosis or vasospasm of the internal auditory artery or of its subdivisions, leads to impaired perfusion of the cochlea. Tissue injury that results from oxygen deprivation and ischaemia can occur in cochlea within 60 seconds. A total but temporary blood circulation blockage can cause damage to the hair cells, the ganglion cells and the spiral ligament and can also cause irreversible events after the blood flow is restored. The labyrinthine artery is extremely vulnerable to blood pressure oscillation and abnormalities in blood flow.

Different treatment regimens for sudden SNHL, such as fibrinogen apheresis, rheopheresis, dextran infusion, HBOT therapy and pentoxifylline have been developed in response to the vascular theory. Zajtchuck et al reported that 8 of 14 patients with sudden SNHL had laboratory evidence of hypercoagulable state manifest as abnormal thrombin generation, increase antithrombin III levels or increased in platelet count $^{8}$. Owen et al found abnormally high levels of antithrombin III levels in one study ${ }^{10}$.Einer et al compared 32 patients with idiopathic sudden SNHL with 28 healthy controls and found that 25 patients had abnormalities in coagulation profile. However, the investigators concluded that pathologic haemostasis did not seem to play a role in the pathogenesis of idiopathic sudden $\mathrm{SNHL}^{14}$. To gain further insight into the mechanisms linking thromboembolism and idiopathic sudden SNHL, we investigated the association between MPV and idiopathic sudden SNHL. Alongside, comparisons were made regarding PDW and PC among cases and healthy controls.MPV values were studied in patients with sudden SNHL yielding controversial results. In recent studies by Ulu et al and by Sagit et al, the MPV values in sudden SNHL patients were $10.5 \pm 0.9 \mathrm{fl}$ and $9.01 \pm 1.24 \mathrm{fl}$ respectively which were higher than the control groups $(9.6 \pm 0.5 \mathrm{fl}$ and $8.21 \pm 0.76 f()^{3,7}$. In another study by Karli et al, (with 46 patients and healthy controls), the MPV was $8.25 \pm 0.86 \mathrm{fl}$ in the cases and $7.98 \pm 0.87 f l$ in the control group, which was not significant. Hence, they concluded that there was no microvascular response in the etiology of sudden $\mathrm{SNHL}^{1}$.

\section{Conclusion}

The indicator for idiopathic sudden SNHL etiology remains a matter of debate because it is associated with many disorders. The pathogenesis of idiopathic sudden SNHL is still unknown although viral infections and vascular occlusions are among the most common mechanisms advocated. Concerning the viral hypothesis, although some studies have been able to demonstrate viral infections in patients with sudden SNHL, the specific and direct pathogenic role of viral infection in inner ear structural 
damage has not yet been definitively proven. In support of the vascular occlusion theory, impaired cochlear perfusion is the widely supported hypothesis although the location of the cochlea in the temporal bone makes identification of a thrombotic occlusion difficult.

In this present study (30 cases and 30 healthy controls), the MPV was $9.37 \pm 1.57 \mathrm{fl}$ in idiopathic sudden SNHL patients and $8.88 \pm 1.35$ fl in the control group with $p$ value being 0.197 . Therefore, based on this value, we concluded that there was no significant difference between the two groups. The absence of any correlation of MPV with any of the studied parameters may suggest that microvascular response is a suspicious theory in the etiopathogenesis of sudden SNHL. In relation to recovery of patients with sudden SNHL, no correlation was observed between MPV levels and patient recovery. However, the results of a study conducted by Ozcan et al indicated that when the idiopathic sudden SNHL patients were observed closely, NLR (neutrophil to lymphocyte ratio) levels were higher in patients who did not recover. This may indicate that as a central role in the etiopathogenesis of idiopathic sudden SNHL, inflammatory events and microvascular blockage remains debatable.

In conclusion, the inferences obtained from this particular study in attempting to establish an association of rise in MPV and other platelet parameters in patients with sudden SNHL, while in agreement with those reported by some previous studies, were in conflict with the results of similar studies by most other groups. Further investigation is required to unequivocally establish the correlation between the two events. It is notable that this study had some strength. The exact selection of patients based on the right inclusion and exclusion criteria and matched healthy controls is the main strength of the study. It would be beneficial to examine the MPV values in further studies with a larger cohort series on patients with idiopathic sudden SNHL.
Conflicts of interest: The authors declare no conflict of interest

\section{References}

1. Anderson RG, Meyerhoff WL. Sudden sensory neural hearing loss. Otolaryngol Clin North Am.1983; 16:189-194

2. Park SN, Yeo SW, Park KH. Serum heat shock proteins 70 and its co-relation with clinical characteristics in patients with sudden sensorineural hearing loss. Laryngoscope. 2006; 116: 121-125

3. M Sagit, M Kavugudurmaz,S Guller,M M Somdas. The journal of laryngology and otology. 2013; 127:972-976

4. Cappacio P, Ottariani F, Cuccarini V, Bottero A, Schindler A, Cesana BM, Censuales S, Pignataro L. Genetic and acquired prothrombotic risk factors and sudden hearing loss. Laryngoscope.2007; 117:547-551

5. Park Y,Schoene N, Harris W. Mean platelet volume as an indicator of platelet activation. Methodological isssues. Platelets. 2002;13: 301-6

6. Gasparyan AY, Avvazyan L, Mikhalidis DP, Kitas GD. Mean platelet volume; A link between thrombosis and inflammation? Curr Pharm Des. 2011; 17910:47-58

7. Yildiz Z, Ulu A, Incesulu A, Ozkaptan Y, Akar N. The importance of thrombotic risk factors in the development of idiopathis sudden sensorineural hearing loss. Clin Appl Thromb Hemost 2008.Jul; 14(3): 356-9

8. Zajtchuk JT, Falor WH Jr, Rhodes MF.Hypercoagulability as a cause of sudden neurosensory hearing loss. Otolaryngol Head Neck Surg. 1979; 87: 268-273

9. Bomholt A, Bak Pederson K, Gormsen J. Fibrinolytic activity in patients with sudden sensorineural hearing loss. Acta 
Otolaryngol Suppl Stockh. 1979; 360:184186

10. Owen MC, Borg JY, Soria J, Caen J, Carell RW. Heparin binding defect in a new antithrombin III variant. Rouen 47, Arg to His Blood. 1987;69: 1275-1279

11. Jaffe BF.Hypercoagulation and other causes of sensorineural hearing loss. Otolaryngol Clin North Am. 1975; 8: 395403

12. Gold S, Kamerer DB, Hirsch BE, Cass SP. Hypercoagulability in otologic patients. Am J Otolaryngol. 1993; 327-331

13. Hudges GB, Fredman MA, Haberkamp TJ, Guay ME. Sudden sensorineural hearing loss.Otolaryngol in North Am. 1996; 29:236-405

14. Einer H,Tengborn L, Axelsson A, Edstrom S. Sudden sensorineural hearing loss. Otolaryngol Head Neck Surg. 1994; 120: 536-40

15. Karli R, Alacam H, Unal R, Kucuk H, Aksoy A, Ayhan E. Mean platelet volume: is it a predictive parameter in the diagnosis of sudden sensorineural hearing loss? Indian J Otolaryngol Head Neck Surg. 2013; 65(4): 350-3. 